\title{
Pourquoi choisir le DES de médecine d'urgence ?
}

\section{Why Choose an Emergency Medicine Diploma?}

\author{
S. Charpentier $\cdot$ F. Dumas \\ (C) SFMU et Lavoisier SAS 2019
}

Chaque année, le choix des étudiants après l'examen classant national (ECN) est un enjeu majeur pour toutes les spécialités [1]. Le nombre de postes non pourvus ainsi que le rang de classement auquel les étudiants choisissent une spécialité peuvent ainsi représenter des indicateurs sur l'attractivité de chacune des disciplines. La création récente du nouveau diplôme d'études spécialisées (DES) de médecine d'urgence implique aussi une réflexion sur l'attrait des étudiants de deuxième et troisième cycles des études médicales pour la discipline.

Les deux articles publiés dans $A F M U[2,3]$ présentent un réel intérêt pour mieux comprendre les éléments et appréhender les leviers potentiels de cette attractivité en médecine d'urgence. Le premier article de Pelaccia et al. [2] a pour but d'identifier les raisons pour lesquelles les étudiants de médecine souhaitent ou non choisir la médecine d'urgence ainsi que leur motivation pour cette spécialité. Dans cet article, l'intérêt des étudiants est dominé par le champ d'activité du métier d'urgentiste comme un facteur d'attractivité constant lors de l'évolution dans le cursus du deuxième cycle, alors que le mode de vie est associé comme paramètre de non-attractivité croissant au décours de leur expérience [2]. Le second article de Douillet et al. [3] évalue le profil motivationnel des étudiants de la première promotion du DES de médecine d'urgence et suggère les leviers et les actions

S. Charpentier $(\square)$

Service des urgences, CHU de Toulouse,

1, avenue Jean-Poulhès, F-31059 Toulouse, France

e-mail : charpentier.s@chu-toulouse.fr

UMR Inserm 1027, faculté de médecine Purpan, université Toulouse-III-Paul-Sabatier,

37, allées Jules-Guesde, F-31000 Toulouse, France

\section{F. Dumas}

Service des urgences,

groupe hospitalier universitaire Paris Centre,

Assistance publique-Hôpitaux de Paris,

27, rue du Faubourg-Saint-Jacques, F-75014 Paris, France

UMR Inserm UMR 970, faculté de médecine, université Paris-Descartes, F-75015 Paris, France potentielles pour les enseignants afin d'améliorer la qualité de la formation. Dans cet article, les auteurs s'intéressent à la motivation intrinsèque des internes pour laquelle l'étudiant va avoir une satisfaction et un intérêt direct à apprendre ce qui a trait à la médecine d'urgence rapportée comme élevée, comparée à la satisfaction extrinsèque (représentation sociale et communautaire de la discipline) [3].

Le premier constat de ces deux études souligne le potentiel d'attractivité de la spécialité par la diversité de situations et le défi intellectuel qu'elle représente, à la fois dans la représentation des étudiants de médecine, mais aussi chez les jeunes internes du DES de médecine d'urgence. Cette observation est primordiale car constante tout au long du cursus de ces étudiants et retrouvée chez les jeunes internes du DES. Cette spécificité est découverte lors des stages cliniques aux urgences et/ou au Samu/SMUR. Cela montre toute l'importance qu'aura la réforme du deuxième cycle qui rendra un stage en médecine d'urgence obligatoire dans le cursus des étudiants hospitaliers. Il y a donc un véritable défi pour les enseignants et encadrants universitaires et non universitaires pour encadrer au mieux les étudiants et leur faire découvrir le potentiel de notre spécialité. Dans un enseignement qui se voulait jusqu'à présent tourné vers la valorisation des spécialités d'organes, l'apprentissage des spécificités des spécialités transversales, dont la médecine d'urgence, est un enjeu majeur pour la spécialité. À ce titre, le Collège national des universitaires de médecine d'urgence s'investit fortement dans la réforme du deuxième cycle des études médicales.

Deuxièmement, ces études mettent en avant les facteurs potentiellement limitant l'attractivité des étudiants, en particulier le mode de vie et la pénibilité perçue. Là aussi, les urgentistes ont un rôle majeur avec la Société française de médecine d'urgence, Samu-Urgences de France et les organisations syndicales pour que la pénibilité d'exercice ne soit pas une fatalité et que des organisations innovantes permettent un exercice de la médecine d'urgence dans des conditions concourant à l'attractivité pour la spécialité. Enfin, nous devons tenir compte du profil des étudiants qui ont choisi la médecine d'urgence actuellement en DES. Cela se traduit par une adaptation de nos méthodes pédagogiques 
avec un apprentissage " actif », une flexibilité et une diversité les lieux de stages, une responsabilisation des internes sur le suivi de leur parcours et de leur évolution.

En conclusion, ces deux évaluations des motivations des étudiants hospitaliers pour la spécialité de médecine d'urgence du début de leur formation aux premières promotions de DES reflètent les aspects riches et attractifs de la spécialité. Elles mettent également en exergue les modalités d'exercice et leur pénibilité perçues par les étudiants comme limitantes dans leur choix. Toutefois, ce constat ne doit pas être présenté comme une fatalité. D'une part, ces observations sont focalisées sur le DES de médecine d'urgence et ne sont pas comparatives avec les autres disciplines médicales qui rencontrent aussi les mêmes problématiques. D'autre part, le DES de médecine d'urgence est naissant et présente une opportunité unique d'évolution à venir si l'accent est porté sur les attentes de ces nouvelles générations. Il appar- tient à la communauté des urgentistes dans son ensemble de rester vigilante, de s'adapter et de répondre proactivement à ses souhaits et à ses craintes.

Liens d'intérêts : les auteurs déclarent ne pas avoir de liens d'intérêts.

\section{Références}

1. Hausfater P (2017) DES de médecine d'urgence aux ECN 2017 : tous les postes pourvus! Oui mais... Ann Fr Med Urgence 7:363-4

2. Pelaccia T, Roy PM, Douillet D, et al (2019) Qu'est-ce qui motive (ou non) les étudiants en médecine à choisir comme spécialité la médecine d'urgence? Ann Fr Med Urgence 9:221-8

3. Douillet D, Ammirati C, Amsallem C, et al (2019) Quelle est la motivation des internes de la première promotion du DES de médecine d'urgence? Ann Fr Med Urgence 9:213-20 